\title{
OPEN Fatty liver index is independently associated with deterioration of renal function during a 10-year period in healthy subjects
}

Satoko Takahashi ${ }^{1,4}$, Marenao Tanaka $^{1,4}$, Masato Furuhashi ${ }^{1 凶}$, Norihito Moniwa ${ }^{1}$, Masayuki Koyama ${ }^{1,2}$, Yukimura Higashiura ${ }^{1}$, Arata Osanami ${ }^{1}$, Yufu Gocho ${ }^{1}$, Hirofumi Ohnishi ${ }^{1,2}{ }^{2}$, Keita Numata ${ }^{3}$, Takashi Hisasue ${ }^{3}$, Nagisa Hanawa ${ }^{3}$ \& Tetsuji Miura ${ }^{1}$

A potential link between chronic kidney disease (CKD) and non-alcoholic fatty liver disease (NAFLD) has been suggested. We investigated the relationship between fatty liver index (FLI), a noninvasive and simple predictor of NAFLD, and the development of CKD defined as estimated glomerular filtration rate $<60 \mathrm{~mL} / \mathrm{min} / 1.73 \mathrm{~m}^{2}$ or positive for urinary protein during a 10-year follow-up period in subjects who received annual health examinations $(n=28,890)$. After exclusion of CKD at baseline, a total of 14,163 subjects (male/female: 9077/5086) were recruited. During the 10-year period, 1458 males (16.1\%) and 737 females (14.5\%) had new onset of CKD. Multivariable Cox proportional hazard models with a restricted cubic spline showed that hazard ratios (HRs) of CKD development increased with a higher FLI at baseline in both males and females after adjustment of confounders. When divided by tertiles of FLI level at baseline (T1 T3), the adjusted risk of CKD development in the T3 group (HR [95\% confidence interval], male/female: 1.33 [1.16-1.54]/1.33 [1.08-1.63]) was significantly higher than that in both sexes in the T1 group as the reference. The addition of FLI into traditional risk factors significantly improved the discriminatory capability for predicting CKD. In conclusion, a high level of FLI predicts the development of CKD in both sexes in a general population.

Chronic kidney disease (CKD) has been recognized as a global public health problem ${ }^{1}$. Previous studies showed that about $10-15 \%$ of the adult population in developed countries suffers from CKD, and the prevalence of $\mathrm{CKD}$ is expected to increase further in the future due to increases in the elderly population and individuals with obesity and diabetes mellitus ${ }^{2,3}$. CKD not only precedes end-stage renal failure but also increases the risks of cardiovascular events and death even in early stages of $\mathrm{CKD}^{1,4}$. Therefore, comprehensive management of risk factors for CKD is clearly a current public need.

Non-alcoholic fatty liver disease (NAFLD) is associated with lifestyle-related diseases such as obesity and metabolic syndrome $e^{5,6}$. It has recently been proposed that liver disease associated with known metabolic dysfunction is newly defined as metabolic dysfunction-associated fatty liver disease (MAFLD) ${ }^{7}$. NAFLD has also been attracting attention as a cause of liver cirrhosis and hepatocellular carcinoma ${ }^{8,9}$. NAFLD is diagnosed in approximately $10-30 \%$ of adults by health examinations ${ }^{10}$, and the number of patients with NAFLD has recently been increasing ${ }^{11}$. It has been reported that NAFLD is a risk factor for insulin resistance, type 2 diabetes mellitus and cardiovascular disease ${ }^{12,13}$. Although diagnosis of NAFLD requires liver biopsy ${ }^{14}$, several noninvasive procedures have recently been established using liver ultrasonography, computed tomography, magnetic resonance spectroscopy and several biochemical indices including fatty liver index (FLI $)^{15}$. FLI, which is calculated by using body mass index (BMI), waist circumference (WC) and levels of $\gamma$-glutamyl transferase $(\gamma$-GTP) and triglycerides, is a noninvasive and simple biomarker for diagnosis of NAFLD ${ }^{16}$ and has a high concordance with the histological criteria for NAFLD ${ }^{17-19}$. This noninvasive index enables analysis of the roles of NAFLD in various diseases using a large group of study subjects.

${ }^{1}$ Department of Cardiovascular, Renal and Metabolic Medicine, Sapporo Medical University School of Medicine, S-1, W-16, Chuo-ku, Sapporo 060-8543, Japan. ${ }^{2}$ Department of Public Health, Sapporo Medical University School of Medicine, Sapporo, Japan. ${ }^{3}$ Department of Health Checkup and Promotion, Keijinkai Maruyama Clinic, Sapporo, Japan. ${ }^{4}$ These authors contributed equally: Satoko Takahashi and Marenao Tanaka. ${ }^{\square}$ email: furuhasi@ sapmed.ac.jp 


\begin{tabular}{|c|c|c|c|c|}
\hline & Total & Male & Female & \\
\hline & $n=14,163$ & $\mathrm{n}=9077$ & $n=5086$ & $\mathbf{P}$ \\
\hline Age (years) & $47 \pm 10$ & $48 \pm 10$ & $46 \pm 10$ & $<0.001$ \\
\hline Body mass index & $23 \pm 3$ & $24 \pm 3$ & $22 \pm 3$ & $<0.001$ \\
\hline Waist circumference $(\mathrm{cm})$ & $83 \pm 9$ & $86 \pm 8$ & $79 \pm 9$ & $<0.001$ \\
\hline Systolic blood pressure (mmHg) & $116 \pm 16$ & $119 \pm 16$ & $110 \pm 16$ & $<0.001$ \\
\hline Diastolic blood pressure $(\mathrm{mmHg})$ & $74 \pm 11$ & $77 \pm 11$ & $69 \pm 10$ & $<0.001$ \\
\hline Smoking habit & $4902(36.2)$ & $3983(45.9)$ & $919(18.9)$ & $<0.001$ \\
\hline Alcohol drinking habit & $6423(45.4)$ & $5122(56.4)$ & $1301(25.6)$ & $<0.001$ \\
\hline \multicolumn{5}{|l|}{ Comorbidity } \\
\hline Hypertension & $2274(16.1)$ & $1760(19.4)$ & $514(10.1)$ & $<0.001$ \\
\hline Diabetes mellitus & $657(4.6)$ & $559(6.2)$ & $98(1.9)$ & $<0.001$ \\
\hline Dyslipidemia & $3226(22.8)$ & $2003(22.1)$ & $1223(24.0)$ & 0.008 \\
\hline \multicolumn{5}{|l|}{ Biochemical data } \\
\hline Hemoglobin $(\mathrm{g} / \mathrm{dL})$ & $14.3 \pm 1.5$ & $15.1 \pm 1.1$ & $12.9 \pm 1.2$ & $<0.001$ \\
\hline Albumin $(\mathrm{g} / \mathrm{dL})$ & $4.4 \pm 0.2$ & $4.4 \pm 0.2$ & $4.3 \pm 0.2$ & $<0.001$ \\
\hline Blood urea nitrogen $(\mathrm{mg} / \mathrm{dL})$ & $14.0 \pm 3.3$ & $14.5 \pm 3.2$ & $13.1 \pm 3.2$ & $<0.001$ \\
\hline Creatinine (mg/dL) & $0.72 \pm 0.14$ & $0.79 \pm 0.10$ & $0.59 \pm 0.08$ & $<0.001$ \\
\hline eGFR $\left(\mathrm{mL} / \mathrm{min} / 1.73 \mathrm{~m}^{2}\right)$ & $86.2 \pm 13.9$ & $85.1 \pm 13.3$ & $88.2 \pm 14.8$ & $<0.001$ \\
\hline Uric acid (mg/dL) & $5.4 \pm 1.4$ & $6.0 \pm 1.2$ & $4.4 \pm 0.9$ & $<0.001$ \\
\hline AST (U/L) & $21(18-26)$ & $22(19-27)$ & $19(16-22)$ & $<0.001$ \\
\hline $\operatorname{ALT}(\mathrm{U} / \mathrm{L})$ & $21(15-31)$ & $25(18-36)$ & $15(12-20)$ & $<0.001$ \\
\hline$\gamma$-GTP (U/L) & $30(19-55)$ & $41(27-72)$ & $18(14-27)$ & $<0.001$ \\
\hline $\mathrm{FPG}(\mathrm{mg} / \mathrm{dL})$ & $93 \pm 18$ & $95 \pm 20$ & $87 \pm 13$ & $<0.001$ \\
\hline Hemoglobin Alc (\%) & $5.3 \pm 0.7$ & $5.4 \pm 0.7$ & $5.2 \pm 0.5$ & $<0.001$ \\
\hline LDL cholesterol (mg/dL) & $121 \pm 31$ & $123 \pm 31$ & $117 \pm 31$ & $<0.001$ \\
\hline HDL cholesterol (mg/dL) & $61 \pm 16$ & $56 \pm 14$ & $69 \pm 15$ & $<0.001$ \\
\hline Triglycerides (mg/dL) & $91(62-136)$ & $109(77-159)$ & $66(49-92)$ & $<0.001$ \\
\hline FLI & $22(8-48)$ & $34(16-58)$ & $7(3-17)$ & $<0.001$ \\
\hline
\end{tabular}

Table 1. Characteristics of the recruited subjects. Variables are expressed as number (\%), means \pm SD or medians (interquartile ranges). AST aspartate aminotransferase, ALT alanine aminotransferase, eGFR estimated glomerular filtration rate, FLI fatty liver index, FPG fasting plasma glucose, $\gamma$-GTP $\gamma$-glutamyl transferase, $H D L$ high-density lipoprotein, $L D L$ low-density lipoprotein.

It has recently been reported that NAFLD diagnosed by liver biopsy, ultrasonography or altered serum liver enzymes is associated with the prevalence and incidence of $\mathrm{CKD}^{20,21}$. To the best of our knowledge, there have been only three studies on the association between FLI and CKD ${ }^{22-24}$. Cross-sectional studies showed that FLI level was associated with decreased estimated glomerular filtration rate (eGFR), increased urinary albumin excretion and prevalence of $\mathrm{CKD}^{22,23}$. A longitudinal study using 4761 Korean subjects (male/female: 1808/2953) also showed that the risk of cumulative incidence of CKD during a 10-year follow-up period was higher in the high FLI group (FLI $\geq 60$ ), a group diagnosed as NAFLD, than in the low FLI group (FLI $<30$ ), a group diagnosed as non-NAFLD ${ }^{24}$. However, despite the fact that there is a sex difference in the level of FLI ${ }^{18}$, there have been no study in which the relationship between FLI level and CKD divided by sex was investigated ${ }^{22-24}$. Furthermore, the possibility of racial difference in cutoff levels of FLI for diagnosis of NAFLD ${ }^{17-19}$ cannot be excluded, and it is not clear whether categorization of FLI using FLI $\geq 60$ or FLI $<30$ is optimal. Therefore, the association between FLI and CKD has not fully been characterized. Considering the uncertainties in earlier studies ${ }^{22-24}$, in the present study, we investigated the relationships of FLI value as a continuous variable and categorized tertile subgroups with the development of CKD during a 10-year follow-up period in a large number of subjects divided by sex.

\section{Results}

Characteristics of the study subjects. Basal characteristics of the recruited subjects are shown in Table 1. Components of FLI calculation, including BMI, WC, $\gamma$-GTP and triglycerides, were significantly higher in male subjects than in female subjects. FLI level was significantly higher in male subjects (median [interquartile ranges] 34 [16-58]) than in female subjects (7 [3-17]).

Basal characteristics of male and female subjects divided by subgroups according to tertiles of FLI at baseline are shown in Tables 2 and 3, respectively. Higher tertiles of FLI were accompanied by larger BMI and WC, higher frequencies of habits of alcohol drinking and smoking, hypertension, diabetes mellitus and dyslipidemia, higher levels of systolic and diastolic blood pressures, hemoglobin, platelet, uric acid, aspartate aminotransferase, alanine aminotransferase, $\gamma$-GTP, fasting plasma glucose, hemoglobin A1c, low-density lipoprotein (LDL) cholesterol 


\begin{tabular}{|c|c|c|c|c|}
\hline & $\mathrm{T} 1[0.8-21.9]$ & T2 [22.0-49.9] & T3 [50.0-99.7] & \\
\hline & $n=3072$ & $\mathrm{n}=3002$ & $\mathrm{n}=3003$ & $\mathbf{P}$ \\
\hline Age (years) & $46 \pm 11$ & $49 \pm 9$ & $48 \pm 9$ & $<0.001$ \\
\hline Body mass index & $21 \pm 2$ & $24 \pm 2$ & $26 \pm 3$ & $<0.001$ \\
\hline Waist circumference $(\mathrm{cm})$ & $79 \pm 5$ & $86 \pm 5$ & $93 \pm 7$ & $<0.001$ \\
\hline Systolic blood pressure $(\mathrm{mmHg})$ & $114 \pm 15$ & $119 \pm 15$ & $124 \pm 15$ & $<0.001$ \\
\hline Diastolic blood pressure $(\mathrm{mmHg})$ & $73 \pm 10$ & $77 \pm 10$ & $80 \pm 10$ & $<0.001$ \\
\hline Smoking habit & $1,327(45.2)$ & $1,280(44.5)$ & $1376(48.0)$ & 0.021 \\
\hline Alcohol drinking habit & $1,526(49.7)$ & $1,761(58.7)$ & $1835(61.1)$ & $<0.001$ \\
\hline \multicolumn{5}{|l|}{ Comorbidity } \\
\hline Hypertension & $300(9.8)$ & $587(19.6)$ & $873(29.1)$ & $<0.001$ \\
\hline Diabetes mellitus & $103(3.4)$ & $188(6.3)$ & $268(8.9)$ & $<0.001$ \\
\hline Dyslipidemia & $527(17.2)$ & $667(22.2)$ & $809(26.9)$ & $<0.001$ \\
\hline \multicolumn{5}{|l|}{ Biochemical data } \\
\hline Hemoglobin $(\mathrm{g} / \mathrm{dL})$ & $14.8 \pm 1.0$ & $15.1 \pm 1.0$ & $15.5 \pm 1.0$ & $<0.001$ \\
\hline Platelet $\left(10^{4} / \mu \mathrm{L}\right)$ & $23.0 \pm 4.7$ & $23.6 \pm 4.9$ & $23.6 \pm 5.1$ & $<0.001$ \\
\hline Albumin (g/dL) & $4.4 \pm 0.2$ & $4.4 \pm 0.2$ & $4.4 \pm 0.2$ & $<0.001$ \\
\hline Blood urea nitrogen $(\mathrm{mg} / \mathrm{dL})$ & $14.5 \pm 3.4$ & $14.6 \pm 3.2$ & $14.4 \pm 3.1$ & 0.043 \\
\hline Creatinine $(\mathrm{mg} / \mathrm{dL})$ & $0.78 \pm 0.10$ & $0.79 \pm 0.10$ & $0.79 \pm 0.10$ & $<0.001$ \\
\hline eGFR $\left(\mathrm{mL} / \mathrm{min} / 1.73 \mathrm{~m}^{2}\right)$ & $86.8 \pm 13.2$ & $84.2 \pm 13.2$ & $84.3 \pm 13.3$ & $<0.001$ \\
\hline Uric acid $(\mathrm{mg} / \mathrm{dL})$ & $5.6 \pm 1.1$ & $6.0 \pm 1.2$ & $6.5 \pm 1.3$ & $<0.001$ \\
\hline $\operatorname{AST}(\mathrm{U} / \mathrm{L})$ & $20(17-23)$ & $22(19-26)$ & $27(22-34)$ & $<0.001$ \\
\hline $\operatorname{ALT}(\mathrm{U} / \mathrm{L})$ & $18(15-24)$ & $25(19-33)$ & $37(27-53)$ & $<0.001$ \\
\hline$\gamma$-GTP $(\mathrm{U} / \mathrm{L})$ & $25(20-34)$ & $42(30-61)$ & $77(50-124)$ & $<0.001$ \\
\hline $\mathrm{FPG}(\mathrm{mg} / \mathrm{dL})$ & $91 \pm 18$ & $95 \pm 18$ & $100 \pm 23$ & $<0.001$ \\
\hline Hemoglobin Alc (\%) & $5.2 \pm 0.6$ & $5.3 \pm 0.7$ & $5.5 \pm 0.8$ & $<0.001$ \\
\hline LDL cholesterol (mg/dL) & $115 \pm 28$ & $126 \pm 30$ & $126 \pm 32$ & $<0.001$ \\
\hline HDL cholesterol (mg/dL) & $62 \pm 14$ & $55 \pm 13$ & $51 \pm 11$ & $<0.001$ \\
\hline Triglycerides (mg/dL) & $73(57-94)$ & $112(88-145)$ & $169(126-233)$ & $<0.001$ \\
\hline
\end{tabular}

Table 2. Characteristics of male subjects divided by tertiles of FLI at baseline $(n=9077)$. Variables are expressed as number (\%), means $\pm \mathrm{SD}$ or medians (interquartile ranges). AST aspartate aminotransferase, ALT alanine aminotransferase, $e G F R$ estimated glomerular filtration rate, FLI fatty liver index, FPG fasting plasma glucose, $\gamma$-GTP $\gamma$-glutamyl transferase, $H D L$ high-density lipoprotein, $L D L$ low-density lipoprotein.

and triglycerides and lower level of high-density lipoprotein (HDL) cholesterol in both sexes. In addition, higher tertiles of FLI were accompanied by higher blood urea nitrogen and lower eGFR in female subjects.

Cumulative incidence of new onset of CKD during a follow-up period. The mean follow-up period was 6.3 years (range 1-10 years), and follow-up summation was 88,733 (male/female: 57,223/31,510) personyears. Among the 14,163 recruited subjects, 1458 male subjects (16.1\%) and 737 female subjects (14.5\%) had new onset of CKD during a 10-year period. Cumulative incidence of CKD was $21.1 \%$ (95\% confidence intervals [CI] 20.2-21.9) (male/female 22.0\% [95\% CI 21.0-23.1]/19.3\% [95\% CI 17.9-20.6]).

Impact of FLI level at baseline on development of CKD during a 10-year follow-up period. In all of the subjects, a multivariable Cox proportional hazard model analysis after adjustment of sex, age, eGFR, hemoglobin, uric acid, habits of smoking and alcohol drinking, and diagnosis of hypertension, diabetes mellitus and dyslipidemia at baseline showed that the adjusted hazard ratio (HR) for CKD development in the 3rd (T3) group of FLI (HR 1.31, 95\% CI 1.16-1.47, P<0.001) was significantly higher than that in the 1st tertile (T1) group of FLI as the reference $(\mathrm{P}$ for trend $=0.024)$ (Supplementary Table S1). There was no significant interaction between sex and tertiles of FLI for the development of CKD $(\mathrm{P}=0.420)$. However, when subjects were divided by sex, the distribution patterns of FLI levels were different between males (Fig. 1A) and females (Fig. 1B).

In male subjects, a multivariable Cox proportional hazard model with a restricted cubic spline showed that the risk of CKD development increased with a higher FLI level at baseline after adjustment of age, eGFR, hemoglobin, uric acid, habits of smoking and alcohol drinking, and diagnosis of hypertension, diabetes mellitus and dyslipidemia at baseline (Fig. 1C). When the T1 group of FLI was used as the reference, multivariable Cox proportional hazard model analysis after adjustment of the covariates showed that HR for CKD development in the T3 group of FLI (HR 1.33, 95\% CI 1.16-1.54, P $<0.001$ ) was significantly higher than that in the T1 group of FLI in male subjects (P for trend $<0.001$ ) (Table 4).

In female subjects, a multivariable Cox proportional hazard model with a restricted cubic spline showed that the risk of CKD development after adjustment of age, eGFR, hemoglobin, uric acid, habits of smoking and 


\begin{tabular}{|c|c|c|c|c|}
\hline & $\mathrm{T} 1[0.4-4.2]$ & T2 $[4.3-12.0]$ & T3 [12.1-99.3] & \\
\hline & $\mathrm{n}=1702$ & $n=1681$ & $n=1703$ & $\mathbf{P}$ \\
\hline Age (years) & $41 \pm 9$ & $47 \pm 10$ & $51 \pm 10$ & $<0.001$ \\
\hline Body mass index & $19 \pm 2$ & $21 \pm 2$ & $25 \pm 3$ & $<0.001$ \\
\hline Waist circumference $(\mathrm{cm})$ & $71 \pm 5$ & $78 \pm 5$ & $87 \pm 8$ & $<0.001$ \\
\hline Systolic blood pressure $(\mathrm{mmHg})$ & $104 \pm 13$ & $109 \pm 15$ & $117 \pm 16$ & $<0.001$ \\
\hline Diastolic blood pressure (mmHg) & $65 \pm 9$ & $69 \pm 10$ & $74 \pm 10$ & $<0.001$ \\
\hline Smoking habit & $294(18.2)$ & $288(17.9)$ & $337(20.5)$ & 0.123 \\
\hline Alcohol drinking habit & $374(22.0)$ & $462(27.5)$ & $465(27.3)$ & $<0.001$ \\
\hline \multicolumn{5}{|l|}{ Comorbidity } \\
\hline Hypertension & $48(2.8)$ & $125(7.4)$ & $341(20.0)$ & $<0.001$ \\
\hline Diabetes mellitus & $6(0.4)$ & $19(1.1)$ & $73(4.3)$ & $<0.001$ \\
\hline Dyslipidemia & $334(19.6)$ & $385(22.9)$ & $504(29.6)$ & $<0.001$ \\
\hline \multicolumn{5}{|l|}{ Biochemical data } \\
\hline Hemoglobin $(\mathrm{g} / \mathrm{dL})$ & $12.7 \pm 1.2$ & $12.8 \pm 1.2$ & $13.2 \pm 1.1$ & $<0.001$ \\
\hline Platelet $\left(10^{4} / \mu \mathrm{L}\right)$ & $23.7 \pm 5.0$ & $24.6 \pm 5.4$ & $25.7 \pm 5.7$ & $<0.001$ \\
\hline Albumin $(\mathrm{g} / \mathrm{dL})$ & $4.3 \pm 0.2$ & $4.3 \pm 0.2$ & $4.3 \pm 0.2$ & 0.065 \\
\hline Blood urea nitrogen $(\mathrm{mg} / \mathrm{dL})$ & $12.9 \pm 3.2$ & $13.1 \pm 3.1$ & $13.4 \pm 3.3$ & $<0.001$ \\
\hline Creatinine $(\mathrm{mg} / \mathrm{dL})$ & $0.59 \pm 0.08$ & $0.59 \pm 0.08$ & $0.58 \pm 0.08$ & 0.131 \\
\hline eGFR $\left(\mathrm{mL} / \mathrm{min} / 1.73 \mathrm{~m}^{2}\right)$ & $90.8 \pm 14.7$ & $87.3 \pm 14.7$ & $86.4 \pm 14.6$ & $<0.001$ \\
\hline Uric acid (mg/dL) & $4.0 \pm 0.8$ & $4.3 \pm 0.9$ & $4.8 \pm 1.0$ & $<0.001$ \\
\hline AST (U/L) & $18(16-20)$ & $18(16-21)$ & $20(17-24)$ & $<0.001$ \\
\hline $\operatorname{ALT}(\mathrm{U} / \mathrm{L})$ & $13(11-16)$ & $14(12-18)$ & $19(15-27)$ & $<0.001$ \\
\hline$\gamma$-GTP (U/L) & $14(12-18)$ & $17(14-23)$ & $27(19-42)$ & $<0.001$ \\
\hline $\mathrm{FPG}(\mathrm{mg} / \mathrm{dL})$ & $84 \pm 9$ & $86 \pm 12$ & $92 \pm 16$ & $<0.001$ \\
\hline Hemoglobin Alc (\%) & $5.1 \pm 0.4$ & $5.2 \pm 0.4$ & $5.4 \pm 0.6$ & $<0.001$ \\
\hline LDL cholesterol (mg/dL) & $105 \pm 26$ & $117 \pm 29$ & $130 \pm 31$ & $<0.001$ \\
\hline HDL cholesterol (mg/dL) & $74 \pm 14$ & $71 \pm 14$ & $63 \pm 15$ & $<0.001$ \\
\hline Triglycerides (mg/dL) & $48(39-59)$ & $67(53-82)$ & $98(75-133)$ & $<0.001$ \\
\hline
\end{tabular}

Table 3. Characteristics of female subjects divided by tertiles of FLI at baseline $(n=5086)$. Variables are expressed as number (\%), means $\pm \mathrm{SD}$ or medians (interquartile ranges). AST aspartate aminotransferase, $A L T$ alanine aminotransferase, eGFR estimated glomerular filtration rate, FLI fatty liver index, FPG fasting plasma glucose, $\gamma$-GTP $\gamma$-glutamyl transferase, $H D L$ high-density lipoprotein, $L D L$ low-density lipoprotein.

alcohol drinking, and diagnosis of hypertension, diabetes mellitus and dyslipidemia at baseline increased with higher FLI at baseline (Fig. 1D). The HR after adjustment of the confounders in the T3 group of FLI (HR 1.33, 95\% CI 1.08-1.63, $\mathrm{P}<0.001$ ) was significantly higher than that in the T1 group of FLI in female subjects ( $\mathrm{P}$ for trend $=0.007)($ Table 4$)$.

Discriminatory capacity of the addition of FLI for predicting the development of CKD. The addition of FLI level into traditional risk factors for CKD, including age, levels of eGFR, hemoglobin and uric acid, habits of smoking and alcohol drinking, and presence of hypertension, diabetes mellitus and dyslipidemia, modestly but significantly increased the area under the receiver operating characteristic curve in both males ( 0.657 vs. $0.662, \mathrm{P}=0.042)$ and females $(0.645$ vs. $0.651, \mathrm{P}=0.023)$ (Table 5$)$. The incorporation of FLI level also led to significant improvements of the discriminatory capacity for predicting CKD development in the continuous net reclassification improvement and integrated discrimination improvement in both males and females (Table 5).

\section{Discussion}

The present study showed that FLI level was independently associated with deterioration of renal function during a 10-year period in healthy subjects. Multivariable Cox proportional hazard models with a restricted cubic spline showed that HRs for CKD development after adjustment of traditional risk factors increased with a higher FLI level at baseline in both male and female subjects. In addition, HRs in the T3 group of FLI were significantly higher than those in the T1 group as the reference in both sexes. Furthermore, the addition of FLI into traditional risk factors significantly improved discriminatory capability of regression models for predicting CKD. Since there is a sex difference in data for components of FLI calculation, including BMI, WC, triglycerides and $\gamma$-GTP, being higher in male subjects than in female subjects ${ }^{25-28}$, it is necessary to analyze the FLI value divided by sex. In fact, there was a significant sex difference in FLI level in the present study as well as in a previous study ${ }^{18}$. A longitudinal study using 4761 Korean subjects (male/female: 1808/2953) showed that the HR for development of CKD defined as eGFR $<60 \mathrm{~mL} / \mathrm{min} / 1.73 \mathrm{~m}^{2}$ during a 10 -year follow-up in the NAFLD group 
A

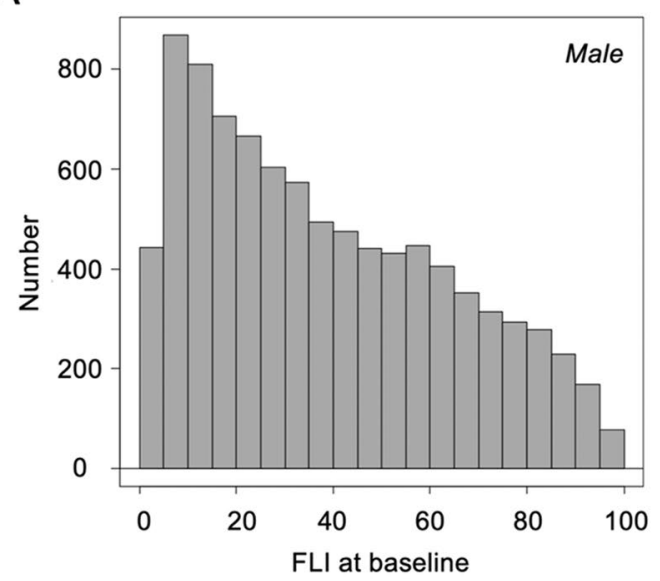

C

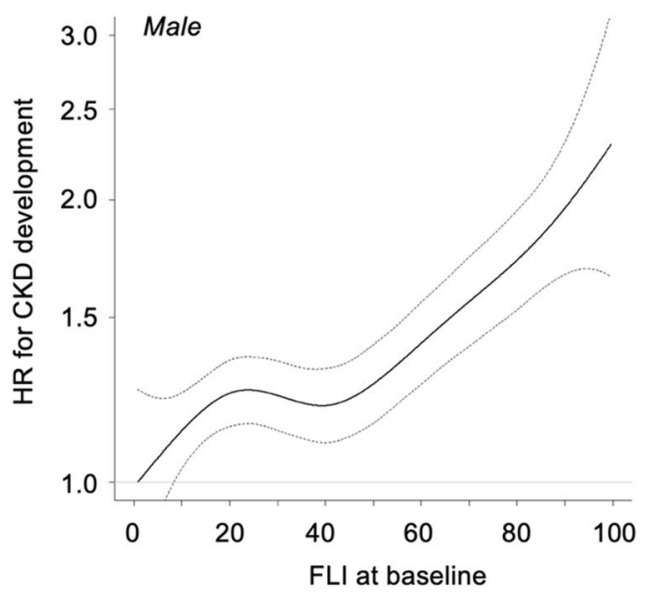

B

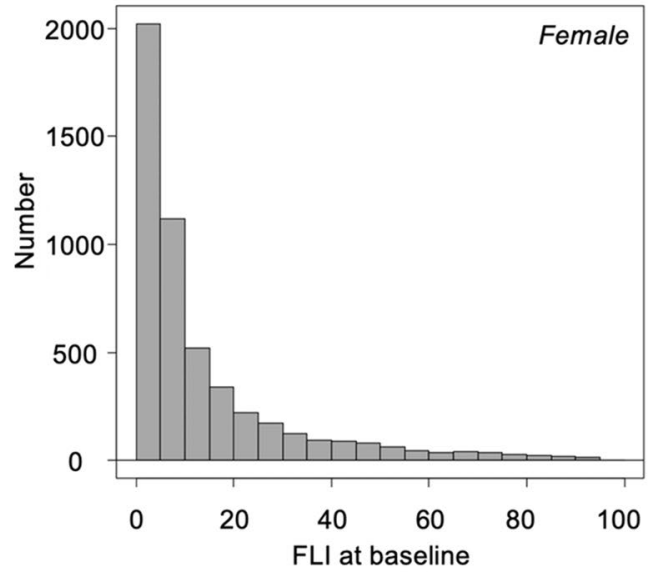

D

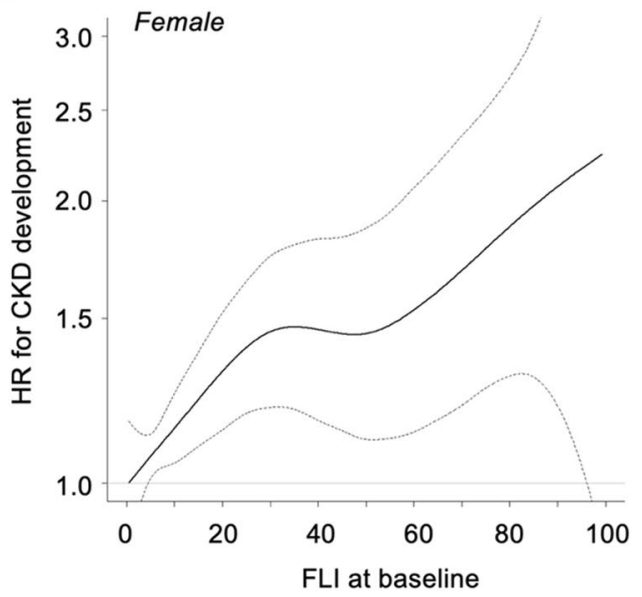

Figure 1. Hazard ratio of the development of CKD by FLI at baseline. (A, B) Histogram of fatty liver index (FLI) at baseline in male subjects (A) and female subjects (B). (C,D) Hazard ratios (HRs) for the development of chronic kidney disease (CKD) by FLI at baseline in male subjects (C) and female subjects (D) analyzed by multivariable Cox proportional hazard models with a restricted cubic spline after adjustment of age, estimated glomerular filtration rate, hemoglobin, uric acid, habits of smoking and alcohol drinking, and diagnosis of hypertension, diabetes mellitus and dyslipidemia at baseline. Solid line: HR; dashed line: $95 \%$ confidence interval. The reference values of FLI in male and female subjects were 0.8 and 0.4 as minimum values, respectively.

$(F L I \geq 60)$ was 1.5 -times higher than that in the non-NAFLD group $(F L I<30)^{24}$. However, the sex difference in FLI level was not taken into consideration in that study ${ }^{24}$. When FLI level is analyzed by both sexes together, the risk for development of CKD might be underestimated in female subjects. It was found in the present study was that both male and female subjects with a high FLI level have an increased risk for the development of CKD and that a high FLI level can predict the development of CKD.

It has been reported that the cutoff level of FLI for diagnosis of NAFLD seems to be lower in Asians than in Europeans: FLI $\geq 30$ in China $^{19}$ and FLI $\geq 60$ in Italy ${ }^{16}$. The subjects were not divided by sex in both of those studies, suggesting that there might be racial and sex differences in cutoff levels of FLI for diagnosis of NAFLD. To the best of our knowledge, the sex difference in the FLI value for diagnosis of NAFLD was considered in only one study performed in Taiwan, and the cutoff levels in male and female subjects in that study were FLI $\geq 35$ and FLI $\geq 25$, respectively ${ }^{18}$. In the present study, most of male (100\%) and female (51\%) subjects in the T3 group of FLI met the FLI criteria for diagnosis of NAFLD (male/female: FLI $\geq 35 / F L I \geq 25$ ) ${ }^{18}$. In female subjects, FLI level might be a risk factor for CKD development regardless of the presence of NAFLD.

There are several possible mechanisms of the link between NAFLD and CKD development. The two diseases share risk factors for metabolic syndrome including obesity, insulin resistance, dyslipidemia and chronic inflammation ${ }^{29,30}$. As other possible mechanisms, a steatotic and inflamed liver has been reported to be a relevant source of proinflammatory, pro-fibrogenic and anti-fibrinolytic molecules including fetuin-A, fibroblast growth factor 2, tumor necrosis factor- $\alpha$, transforming growth factor- $\beta$ and plasminogen activator inhibitor- 1 , which theoretically can promote kidney injury ${ }^{31}$. Furthermore, fatty liver may promote glomerular injury and mesangial cell proliferation through increased secretion of very low-density lipoprotein and induction of atherogenic 


\begin{tabular}{|c|c|c|c|c|c|c|c|c|}
\hline & \multicolumn{4}{|l|}{ Male $(n=9077)$} & \multicolumn{4}{|l|}{ Female $(n=5086)$} \\
\hline & HR (95\% CI) & $\mathbf{P}$ & HR (95\% CI) & $\mathbf{P}$ & HR $(95 \%$ CI $)$ & $\mathbf{P}$ & HR (95\% CI) & $\mathbf{P}$ \\
\hline \multicolumn{9}{|l|}{ FLI } \\
\hline $\mathrm{T} 1$ & Reference & - & Reference & - & Reference & - & Reference & - \\
\hline $\mathrm{T} 2$ & $1.14(1.00-1.30)$ & 0.058 & $1.01(0.88-1.16)$ & 0.900 & $1.16(0.96-1.39)$ & 0.120 & $1.15(0.94-1.39)$ & 0.170 \\
\hline T3 & $1.51(1.33-1.71)$ & $<0.001$ & $1.33(1.16-1.54)$ & $<0.001$ & $1.40(1.18-1.68)$ & $<0.001$ & $1.33(1.08-1.63)$ & 0.007 \\
\hline P for trend & & $<0.001$ & & $<0.001$ & & $<0.001$ & & 0.007 \\
\hline Age (per 10 years) & - & - & $1.04(0.98-1.11)$ & 0.190 & - & - & $0.82(0.75-0.89)$ & $<0.001$ \\
\hline $\begin{array}{l}\text { eGFR }(\text { per } 1 \mathrm{~mL} / \\
\left.\mathrm{min} / 1.73 \mathrm{~m}^{2}\right)\end{array}$ & - & - & $0.96(0.96-0.97)$ & $<0.001$ & - & - & $0.97(0.96-0.97)$ & $<0.001$ \\
\hline $\begin{array}{l}\text { Hemoglobin (per } \\
1 \mathrm{~g} / \mathrm{dL} \text { ) }\end{array}$ & - & - & $0.99(0.94-1.05)$ & 0.780 & - & - & $0.91(0.86-0.97)$ & 0.005 \\
\hline $\begin{array}{l}\text { Uric acid (per } 1 \mathrm{mg} / \\
\text { dL) }\end{array}$ & - & - & $0.98(0.93-1.02)$ & 0.290 & - & - & $1.07(0.98-1.16)$ & 0.150 \\
\hline Smoking habit & - & - & $1.24(1.12-1.39)$ & $<0.001$ & - & - & $1.22(1.01-1.47)$ & 0.042 \\
\hline $\begin{array}{l}\text { Alcohol drinking } \\
\text { habit }\end{array}$ & - & - & $0.81(0.73-0.90)$ & $<0.001$ & - & - & $0.92(0.77-1.09)$ & 0.330 \\
\hline Hypertension & - & - & $1.49(1.32-1.70)$ & $<0.001$ & - & - & $1.58(1.25-1.98)$ & $<0.001$ \\
\hline Diabetes mellitus & - & - & $1.46(1.20-1.77)$ & $<0.001$ & - & - & $1.97(1.28-3.02)$ & 0.002 \\
\hline \multirow[t]{2}{*}{ Dyslipidemia } & - & - & $1.00(0.88-1.13)$ & 0.990 & - & - & $1.15(0.97-1.36)$ & 0.100 \\
\hline & \multicolumn{2}{|l|}{$(\mathrm{AIC}=25,451)$} & \multicolumn{2}{|l|}{$(\mathrm{AIC}=23,989)$} & \multicolumn{2}{|l|}{$(\mathrm{AIC}=12,088)$} & \multicolumn{2}{|l|}{$(\mathrm{AIC}=11,374)$} \\
\hline
\end{tabular}

Table 4. Multivariable Cox proportional hazard analyses for new onset of CKD in tertiles of FLI. AIC Akaike's information criterion, $C I$ confidence interval, $C K D$ chronic kidney disease, $e G F R$ estimated glomerular filtration rate, FLI fatty liver index, HR hazard ratio.

\begin{tabular}{|c|c|c|c|c|c|c|}
\hline & \multicolumn{2}{|l|}{ AUC } & \multicolumn{2}{|l|}{ NRI } & \multicolumn{2}{|l|}{ IDI } \\
\hline & Value (95\% CI) & $\mathbf{P}$ & Value (95\% CI) & $\mathbf{P}$ & Value $(95 \% \mathrm{CI})$ & $\mathbf{P}$ \\
\hline \multicolumn{7}{|l|}{ Males } \\
\hline Traditional model $^{\mathrm{a}}$ & $0.657(0.640-0.674)$ & - & - & - & - & - \\
\hline Traditional model ${ }^{\mathrm{a}}+$ FLI & $0.662(0.645-0.678)$ & 0.042 & $0.135(0.078-0.192)$ & $<0.001$ & $0.004(0.002-0.005)$ & $<0.001$ \\
\hline \multicolumn{7}{|l|}{ Females } \\
\hline Traditional model $^{\mathrm{a}}$ & $0.645(0.621-0.668)$ & - & - & - & - & - \\
\hline Traditional model ${ }^{\mathrm{a}}+$ FLI & $0.651(0.628-0.675)$ & 0.023 & $0.124(0.047-0.201)$ & 0.001 & $0.003(0.001-0.005)$ & 0.001 \\
\hline
\end{tabular}

Table 5. Discrimination of the addition of FLI into traditional risk factors for CKD. AUC area under the curve, $C I$ confidence interval, $C K D$ chronic kidney disease, FLI fatty liver index, IDI integrated discrimination

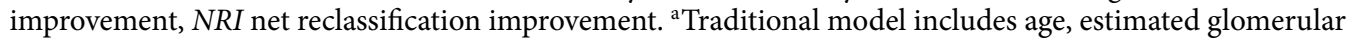
filtration rate, hemoglobin, uric acid, smoking habit, alcohol drinking habit, hypertension, diabetes mellitus and dyslipidemia.

dyslipidemia such as triglycerides-rich lipoproteins and oxidized LDLs ${ }^{31}$. Xanthine oxidoreductase (XOR), a rate-limiting enzyme of uric acid production in the purine metabolism, is abundantly expressed in the liver and can increase reactive oxygen species by generating superoxide and hydrogen peroxide ${ }^{32}$. It has been reported that plasma activity of XOR is a novel biomarker of metabolic disorder ${ }^{33}$ and that change in XOR activity is significantly associated with changes in liver enzymes and body weight ${ }^{34}$. Inadequate activation of XOR in NAFLD may promote oxidative stress-related tissue injury including injury of the kidney ${ }^{32}$.

BMI, WC and triglycerides, components of FLI calculation, have been reported to be risk factors for the development of CKD ${ }^{35,36}$. The other component of FLI, $\gamma$-GTP, also known as $\gamma$-glutamyltransferase, is a cellsurface enzyme and has a physiological role in metabolizing extracellular reduced glutathione, a main antioxidant in mammalian cells ${ }^{37}$. Previous studies showed that elevated $\gamma$-GTP level was a significant predictor of hypertension, diabetes mellitus, cardiovascular disease, congestive heart failure and metabolic syndrome ${ }^{38-41}$. It has also been reported that $\gamma$-GTP level is associated with incidence of CKD in Asian subjects ${ }^{38,42}$, though a meta-analysis showed no significant association between elevated serum $\gamma$-GTP and risk of CKD in an adult general population ${ }^{43}$. Since FLI is an index that includes elements of metabolic syndrome ${ }^{44}$, FLI may represent integrated risk factors for CKD development.

Some interventional studies for improvement of renal function in patients with NAFLD have been carried out $^{45,46}$. Modification of lifestyle improved liver histology and renal function in patients with non-alcoholic steatohepatitis ${ }^{45}$. Progression of CKD was also suppressed by reducing the waist-hip ratio in non-obese patients with NAFLD ${ }^{46}$. Reduction of visceral fat may affect functions of kidney and liver through a decrease in chronic 


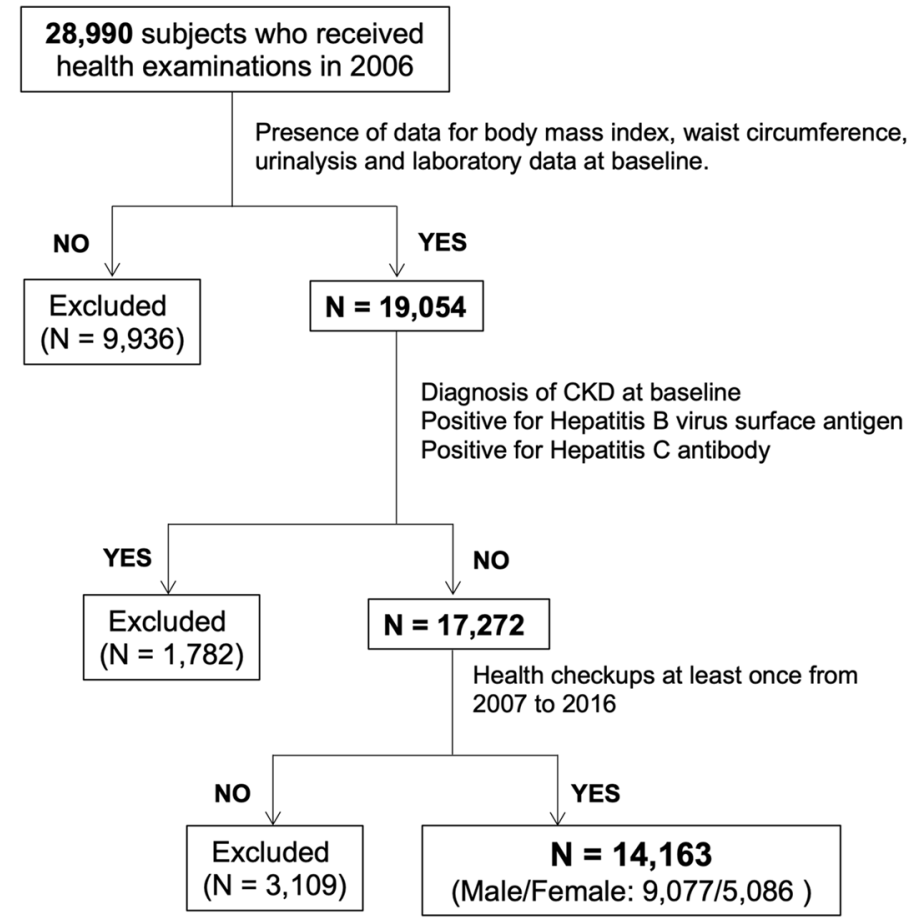

Figure 2. Flow chart of the selected study participants. Among 28,990 subjects enrolled in 2006, a total of 14,163 subjects (male/female: 9077/5086) were finally recruited for analyses in the present study. Chronic kidney disease (CKD) was defined as estimated glomerular filtration rate $(\mathrm{eGFR})<60 \mathrm{~mL} / \mathrm{min} / 1.73 \mathrm{~m}^{2}$ or positive for proteinuria.

low-grade systemic inflammation and suppression of the fibrotic process. Drug development and clinical trials for the treatment of NAFLD are currently being conducted worldwide ${ }^{47}$. Not only modification of conventional risk factors of CKD, which may be related to the common pathogenesis of CKD and NAFLD ${ }^{29,30}$, but also intervention for NAFLD by direct and indirect regulation of hepatokines and/or liver-derived molecules, in addition to modification of conventional CKD risk factors, might contribute to prevention of CKD. Reduction of the FLI level by treatment for NAFLD might be beneficial for prevention of CKD development. Further investigations are needed to determine whether FLI-guided prevention and/or treatment of NAFLD reduces the number of individuals with deterioration of renal function.

The present study has some limitations. First, since the study subjects had a yearly health check-up at a single urban clinic, the possibility of sample selection bias cannot be ruled out. Second, proteinuria was assessed only by the qualitative method since quantitative data for proteinuria were not available. Third, since diagnosis of hepatic steatosis was performed by FLI but not by imaging techniques, the severity of hepatic steatosis was not taken into consideration. Finally, since most of the participants in the present study were middle-aged subjects, the results of the present study may not be directly applicable to elderly subjects. Investigation of the relationship between FLI and CKD development in elderly subjects is needed in the future.

In conclusion, a high level of FLI, originally developed as an indicator of NAFLD, predicts new onset of CKD in both males and females. The addition of FLI into traditional risk factors significantly improves discriminatory capability for prediction of the development CKD. A further understanding of the mechanism of the link between FLI and CKD may enable the development of new therapeutic strategies for prevention of CKD.

\section{Methods}

The present study was conducted as a project of the Broad-range Organization for REnal, Arterial and cardiac studies by Sapporo Medical University Affiliates (BOREAS) investigators and was designed as the BOREASCKD4 study. The study conformed to the principles outlined in the Declaration of Helsinki and was performed with the approval of the institutional ethical committee of Sapporo Medical University (Numbers: 29-2-64, 30-2-32). Written informed consent was obtained from all of the subjects.

Study subjects. All of subjects who received annual health examinations at Keijinkai Maruyama Clinic, Sapporo, Japan in 2006 were enrolled in this registry $(n=28,990)^{48,49}$. A flow chart of the study participants is shown in Fig. 2. Prespecified exclusion criteria were the absence of data for BMI, WC, urinalysis and laboratory data including serum creatinine, eGFR, triglycerides and $\gamma$-GTP at baseline, positive for hepatitis B virus surface antigen or hepatitis $\mathrm{C}$ virus antibody, and diagnosis of CKD at baseline. After exclusion, a total of 14,163 subjects (male/female: 9077/5086) who received health examinations at least once in the period from 2007 to 2016 were 
recruited in the present study. The number of subjects who received annual health checkup at the end of the follow-up period was 5683 (male/female: 3751/1932). A self-administered questionnaire survey was performed to obtain information on smoking habit, alcohol drinking habit and use of drugs for diabetes mellitus, hypertension and dyslipidemia.

Measurements. Medical examinations, blood pressure measurements and samplings of urine and blood were performed after an overnight fast. Body height and weight were measured in light clothing without shoes, and BMI was calculated as body weight in kilograms divided by height in meters squared. As an indicator of renal function, eGFR was calculated by the following equation: eGFR $\left(\mathrm{mL} / \mathrm{min} / 1.73 \mathrm{~m}^{2}\right)=194 \times$ serum creatinine $\mathrm{e}^{(-1.094)} \times$ age $^{(-0.287)} \times 0.739$ (if female) $)^{50}$. FLI was calculated using the algorithm reported by Bedogni et al. ${ }^{16}: \mathrm{FLI}=\left[\mathrm{e}^{(0.953 \times \ln (\text { triglycerides })+0.139 \times \mathrm{BMI}+0.718 \times \ln (\gamma-\mathrm{GTP})+0.053 \times \mathrm{WC}-15.745)}\right] /\left[1+\mathrm{e}^{(0.953 \times \ln (\text { triglycerides })+0.139 \times \mathrm{BMI}+0.718 \times \ln (\gamma-\mathrm{GTP})+0.0}\right.$ $53 \times$ WC-15.745) $] \times 100$.

CKD was defined as eGFR $<60 \mathrm{~mL} / \mathrm{min} / 1.73 \mathrm{~m}^{2}$ or positive for urinary protein by the dipstick method. Diabetes mellitus was diagnosed in accordance with the guideline of the American Diabetes Association ${ }^{51}$ : fasting plasma glucose $\geq 126 \mathrm{mg} / \mathrm{dL}$, hemoglobin $\mathrm{A} 1 \mathrm{c} \geq 6.5 \%$ or self-reported use of anti-diabetic drugs. Hypertension was diagnosed as systolic blood pressure $\geq 140 \mathrm{mmHg}$, diastolic blood pressure $\geq 90 \mathrm{mmHg}$ or self-reported use of anti-hypertensive drugs. Dyslipidemia was diagnosed as LDL cholesterol $\geq 140 \mathrm{mg} / \mathrm{dL}$, HDL cholesterol $<40 \mathrm{mg} /$ $\mathrm{dL}$, triglycerides $\geq 150 \mathrm{mg} / \mathrm{dL}$ or self-reported use of anti-dyslipidemic drugs.

Statistical analysis. Numeric variables are expressed as means \pm SD for parameters with normal distributions and as medians (interquartile ranges) for parameters with skewed distributions. The distribution of each parameter was tested for its normality using the Shapiro-Wilk W test. Comparisons between two groups for parametric and nonparametric parameters were performed by using Student's t-test and the Mann-Whitney U test, respectively. Clinical parameters were divided into three subgroups according to tertiles of FLI at baseline (T1 T3) in both male and female subjects. Intergroup differences in percentages of demographic parameters were examined by the chi-square test. One-way analysis of variance for parametric parameters and the KruskalWallis test for nonparametric parameters were used for detecting significant differences in data between multiple groups. The association between FLI levels at baseline and the development of CKD was investigated by multivariable Cox proportional hazard models with a restricted cubic spline after adjustment of confounders including age, eGFR, hemoglobin, uric acid, smoking habit, alcohol drinking habit and diagnosis of hypertension, diabetes mellitus and dyslipidemia at baseline. In addition, HRs and 95\% CIs for the development of CKD in tertiles of FLI level at baseline were calculated by adjustment of the confounders. To compare the discrimination of CKD development between the models adjusted for confounders as traditional risk factors for CKD with and without FLI level, C-statistics analogous to the area under the receiver operating characteristic curve were estimated using the method of DeLong et al. $^{52}$. Moreover, the increased discriminatory value of FLI level was examined by the continuous net reclassification improvement and integrated discrimination improvement ${ }^{53}$. A p value of less than 0.05 was considered statistically significant. All data were analyzed by using EZR ${ }^{54}$ and $\mathrm{R}$ version 3.6.1.

\section{Data availability}

The datasets analyzed during the current study are available from the corresponding author on reasonable request.

Received: 11 November 2020; Accepted: 5 April 2021

Published online: 21 April 2021

\section{References}

1. Matsushita, K. et al. Association of estimated glomerular filtration rate and albuminuria with all-cause and cardiovascular mortality in general population cohorts: A collaborative meta-analysis. Lancet 375, 2073-2081. https://doi.org/10.1016/S0140-6736(10) 60674-5 (2010).

2. Coresh, J. et al. Prevalence of chronic kidney disease in the United States. JAMA 298, 2038-2047. https://doi.org/10.1001/jama. 298.17.2038 (2007).

3. Imai, E. et al. Prevalence of chronic kidney disease in the Japanese general population. Clin. Exp. Nephrol. 13, 621-630. https:// doi.org/10.1007/s10157-009-0199-x (2009).

4. Hallan, S. I. et al. Age and association of kidney measures with mortality and end-stage renal disease. JAMA 308, 2349-2360. https://doi.org/10.1001/jama.2012.16817 (2012).

5. Marchesini, G. et al. Nonalcoholic fatty liver, steatohepatitis, and the metabolic syndrome. Hepatology 37, 917-923. https://doi. org/10.1053/jhep.2003.50161 (2003).

6. Hamaguchi, M. et al. The metabolic syndrome as a predictor of nonalcoholic fatty liver disease. Ann. Intern. Med. 143, 722-728. https://doi.org/10.7326/0003-4819-143-10-200511150-00009 (2005).

7. Eslam, M. et al. A new definition for metabolic dysfunction-associated fatty liver disease: An international expert consensus statement. J. Hepatol. 73, 202-209. https://doi.org/10.1016/j.jhep.2020.03.039 (2020).

8. Bhala, N. et al. The natural history of nonalcoholic fatty liver disease with advanced fibrosis or cirrhosis: An international collaborative study. Hepatology 54, 1208-1216. https://doi.org/10.1002/hep.24491 (2011).

9. Baffy, G., Brunt, E. M. \& Caldwell, S. H. Hepatocellular carcinoma in non-alcoholic fatty liver disease: An emerging menace. J. Hepatol. 56, 1384-1391. https://doi.org/10.1016/j.jhep.2011.10.027 (2012).

10. Amarapurkar, D. N. et al. How common is non-alcoholic fatty liver disease in the Asia-Pacific region and are there local differences?. J. Gastroenterol. Hepatol. 22, 788-793. https://doi.org/10.1111/j.1440-1746.2007.05042.x (2007).

11. Younossi, Z. M. et al. Global epidemiology of nonalcoholic fatty liver disease-meta-analytic assessment of prevalence, incidence, and outcomes. Hepatology 64, 73-84. https://doi.org/10.1002/hep.28431 (2016).

12. Ong, J. P., Pitts, A. \& Younossi, Z. M. Increased overall mortality and liver-related mortality in non-alcoholic fatty liver disease. J. Hepatol. 49, 608-612. https://doi.org/10.1016/j.jhep.2008.06.018 (2008) 
13. Souza, M. R., Diniz Mde, F., Medeiros-Filho, J. E. \& Araujo, M. S. Metabolic syndrome and risk factors for non-alcoholic fatty liver disease. Arq. Gastroenterol. 49, 89-96. https://doi.org/10.1590/s0004-28032012000100015 (2012).

14. Neuschwander-Tetri, B. A. \& Caldwell, S. H. Nonalcoholic steatohepatitis: Summary of an AASLD Single Topic Conference. Hepatology 37, 1202-1219 (2003).

15. Papagianni, M., Sofogianni, A. \& Tziomalos, K. Non-invasive methods for the diagnosis of nonalcoholic fatty liver disease. World J. Hepatol. 7, 638-648. https://doi.org/10.4254/wjh.v7.i4.638 (2015).

16. Bedogni, G. et al. The Fatty Liver Index: A simple and accurate predictor of hepatic steatosis in the general population. BMC Gastroenterol. 6, 33. https://doi.org/10.1186/1471-230X-6-33 (2006).

17. Otgonsuren, M. et al. Single non-invasive model to diagnose non-alcoholic fatty liver disease (NAFLD) and non-alcoholic steatohepatitis (NASH). J. Gastroenterol. Hepatol. 29, 2006-2013. https://doi.org/10.1111/jgh.12665 (2014).

18. Yang, B. L. et al. External validation of fatty liver index for identifying ultrasonographic fatty liver in a large-scale cross-sectional study in Taiwan. PLoS ONE 10, e0120443. https://doi.org/10.1371/journal.pone.0120443 (2015).

19. Huang, X. et al. Validation of the fatty liver index for nonalcoholic fatty liver disease in middle-aged and elderly Chinese. Medicine (Baltimore) 94, e1682. https://doi.org/10.1097/MD.0000000000001682 (2015).

20. Targher, G., Chonchol, M. B. \& Byrne, C. D. CKD and nonalcoholic fatty liver disease. Am. J. Kidney Dis. 64, 638-652. https://doi. org/10.1053/j.ajkd.2014.05.019 (2014).

21. Mantovani, A. et al. Nonalcoholic fatty liver disease increases risk of incident chronic kidney disease: A systematic review and meta-analysis. Metabolism 79, 64-76. https://doi.org/10.1016/j.metabol.2017.11.003 (2018).

22. Zeng, J. et al. Association between non-invasively diagnosed hepatic steatosis and chronic kidney disease in Chinese adults on their health check-up. J. Dig. Dis 18, 229-236. https://doi.org/10.1111/1751-2980.12465 (2017).

23. Sun, K. et al. Fatty liver index, albuminuria and the association with chronic kidney disease: A population-based study in China. BMJ Open 8, e019097. https://doi.org/10.1136/bmjopen-2017-019097 (2018).

24. Huh, J. H. et al. The fatty liver index as a predictor of incident chronic kidney disease in a 10-year prospective cohort study. PLoS ONE 12, e0180951. https://doi.org/10.1371/journal.pone.0180951 (2017).

25. Examination Committee of Criteria for "Obesity Disease" in Japan. New criteria for "obesity disease" in Japan. Circ. J. 66, 987-992. https://doi.org/10.1253/circj.66.987 (2002).

26. Satoh, H., Kishi, R. \& Tsutsui, H. Body mass index can similarly predict the presence of multiple cardiovascular risk factors in middle-aged Japanese subjects as waist circumference. Intern. Med. 49, 977-982. https://doi.org/10.2169/internalmedicine.49. 3006 (2010).

27. Nakamura, K. et al. Optimal cutoff values of waist circumference and the discriminatory performance of other anthropometric indices to detect the clustering of cardiovascular risk factors for metabolic syndrome in Japanese men and women. Environ. Health Prev. Med. 16, 52-60. https://doi.org/10.1007/s12199-010-0165-y (2011).

28. Unno, M. et al. The utility of visceral fat level by bioelectrical impedance analysis in the screening of metabolic syndrome-the results of the Kyushu and Okinawa Population Study (KOPS). J. Atheroscler. Thromb. 19, 462-470. https://doi.org/10.5551/jat. 11528 (2012).

29. Rivera, C. A. Risk factors and mechanisms of non-alcoholic steatohepatitis. Pathophysiology 15, 109-114. https://doi.org/10.1016/j. pathophys.2008.04.003 (2008).

30. Targher, G., Chonchol, M., Zoppini, G., Abaterusso, C. \& Bonora, E. Risk of chronic kidney disease in patients with non-alcoholic fatty liver disease: Is there a link?. J. Hepatol. 54, 1020-1029. https://doi.org/10.1016/j.jhep.2010.11.007 (2011).

31. Musso, G. et al. Association of non-alcoholic fatty liver disease with chronic kidney disease: A systematic review and meta-analysis. PLoS Med. 11, e1001680. https://doi.org/10.1371/journal.pmed.1001680 (2014).

32. Furuhashi, M. New insights into purine metabolism in metabolic diseases: Role of xanthine oxidoreductase activity. Am. J. Physiol. Endocrinol. Metab. 319, E827-E834. https://doi.org/10.1152/ajpendo.00378.2020 (2020).

33. Furuhashi, M. et al. Plasma xanthine oxidoreductase activity as a novel biomarker of metabolic disorders in a general population. Circ. J. 82, 1892-1899. https://doi.org/10.1253/circj.CJ-18-0082 (2018).

34. Furuhashi, M. et al. Annual change in plasma xanthine oxidoreductase activity is associated with changes in liver enzymes and body weight. Endocr. J. 66, 777-786. https://doi.org/10.1507/endocrj.EJ19-0053 (2019).

35. Hsu, C. Y., McCulloch, C. E., Iribarren, C., Darbinian, J. \& Go, A. S. Body mass index and risk for end-stage renal disease. Ann. Intern. Med. 144, 21-28. https://doi.org/10.7326/0003-4819-144-1-200601030-00006 (2006).

36. Noori, N., Hosseinpanah, F., Nasiri, A. A. \& Azizi, F. Comparison of overall obesity and abdominal adiposity in predicting chronic kidney disease incidence among adults. J. Ren. Nutr. 19, 228-237. https://doi.org/10.1053/j.jrn.2008.11.005 (2009).

37. Whitfield, J. B. Gamma glutamyl transferase. Crit. Rev. Clin. Lab. Sci. 38, 263-355. https://doi.org/10.1080/20014091084227 (2001).

38. Shen, Z. W. et al. Association between serum gamma-glutamyltransferase and chronic kidney disease in urban Han Chinese: A prospective cohort study. Int. Urol. Nephrol. 49, 303-312. https://doi.org/10.1007/s11255-016-1429-2 (2017).

39. Jimba, S. et al. Increase in gamma-glutamyltransferase level and development of established cardiovascular risk factors and diabetes in Japanese adults. Metab. Syndr. Relat. Disord. 7, 411-418. https://doi.org/10.1089/met.2008.0082 (2009).

40. Kim, K. M. et al. Serum gamma-glutamyltransferase as a risk factor for general cardiovascular disease prediction in Koreans. J. Investig. Med. 60, 1199-1203. https://doi.org/10.2310/JIM.0b013e3182746752 (2012).

41. Han, K. S., Cho, D. Y., Kim, Y. S. \& Kim, K. N. Serum gamma-glutamyl transferase concentration within the reference range is related to the coronary heart disease risk prediction in Korean men: Analysis of the Korea National Health and Nutrition Examination Survey (V-1, 2010 and V-2, 2011). Chin. Med. J. (Engl.) 128, 2006-2011. https://doi.org/10.4103/0366-6999.161343 (2015).

42. Ryu, S., Chang, Y., Kim, D. I., Kim, W. S. \& Suh, B. S. Gamma-Glutamyltransferase as a predictor of chronic kidney disease in nonhypertensive and nondiabetic Korean men. Clin. Chem. 53, 71-77. https://doi.org/10.1373/clinchem.2006.078980 (2007).

43. Fan, Y., Jin, X., Man, C. \& Gong, D. Association of serum gamma-glutamyltransferase with chronic kidney disease risk: A metaanalysis. Free Radic. Res. 52, 819-825. https://doi.org/10.1080/10715762.2018.1492120 (2018).

44. Perez-Martinez, P. et al. Lifestyle recommendations for the prevention and management of metabolic syndrome: An international panel recommendation. Nutr. Rev. 75, 307-326. https://doi.org/10.1093/nutrit/nux014 (2017).

45. Vilar-Gomez, E. et al. Improvement in liver histology due to lifestyle modification is independently associated with improved kidney function in patients with non-alcoholic steatohepatitis. Aliment Pharmacol. Ther. 45, 332-344. https://doi.org/10.1111/ apt.13860 (2017)

46. Chon, Y. E. et al. Decrease in waist-to-hip ratio reduced the development of chronic kidney disease in non-obese non-alcoholic fatty liver disease. Sci. Rep. 10, 8996. https://doi.org/10.1038/s41598-020-65940-y (2020).

47. Sumida, Y. \& Yoneda, M. Current and future pharmacological therapies for NAFLD/NASH. J. Gastroenterol. 53, 362-376. https:// doi.org/10.1007/s00535-017-1415-1 (2018).

48. Higashiura, Y. et al. Low urine $\mathrm{pH}$ predicts new onset of diabetes mellitus during a 10-year period in men: BOREAS-DM1 study. J. Diabetes Investig. https://doi.org/10.1111/jdi.13284 (2020).

49. Mori, K. et al. U-shaped relationship between serum uric acid level and decline in renal function during a 10 -year period in female subjects: BOREAS-CKD2. Hypertens. Res. https://doi.org/10.1038/s41440-020-0532-z (2020).

50. Matsuo, S. et al. Revised equations for estimated GFR from serum creatinine in Japan. Am. J. Kidney Dis. 53, 982-992. https://doi. org/10.1053/j.ajkd.2008.12.034 (2009). 
51. American Diabetes Association. 2. Classification and diagnosis of diabetes. Diabetes Care 40, S11-S24. https://doi.org/10.2337/ dc17-S005 (2017).

52. DeLong, E. R., DeLong, D. M. \& Clarke-Pearson, D. L. Comparing the areas under two or more correlated receiver operating characteristic curves: A nonparametric approach. Biometrics 44, 837-845 (1988).

53. Pencina, M. J., D’Agostino, R. B. Sr. \& Steyerberg, E. W. Extensions of net reclassification improvement calculations to measure usefulness of new biomarkers. Stat. Med. 30, 11-21. https://doi.org/10.1002/sim.4085 (2011).

54. Kanda, Y. Investigation of the freely available easy-to-use software "EZR" for medical statistics. Bone Marrow Transplant. 48, 452-458. https://doi.org/10.1038/bmt.2012.244 (2013).

\section{Author contributions}

S.T., M.T., and M.F. designed the study, performed data analyses and wrote the paper. N.M., Y.H., A.O., and Y.G. performed data analyses. K.N., T.H., and N.H. performed data collection. M.K. and H.O. performed the statistical analyses. T.M. supervised the analyses. All authors approved the final version of manuscript.

\section{Competing interests}

The authors declare no competing interests.

\section{Additional information}

Supplementary Information The online version contains supplementary material available at https://doi.org/ 10.1038/s41598-021-88025-w.

Correspondence and requests for materials should be addressed to M.F.

Reprints and permissions information is available at www.nature.com/reprints.

Publisher's note Springer Nature remains neutral with regard to jurisdictional claims in published maps and institutional affiliations.

(c) (1) Open Access This article is licensed under a Creative Commons Attribution 4.0 International License, which permits use, sharing, adaptation, distribution and reproduction in any medium or format, as long as you give appropriate credit to the original author(s) and the source, provide a link to the Creative Commons licence, and indicate if changes were made. The images or other third party material in this article are included in the article's Creative Commons licence, unless indicated otherwise in a credit line to the material. If material is not included in the article's Creative Commons licence and your intended use is not permitted by statutory regulation or exceeds the permitted use, you will need to obtain permission directly from the copyright holder. To view a copy of this licence, visit http://creativecommons.org/licenses/by/4.0/.

(C) The Author(s) 2021 